\title{
Guided Botulinum Toxin Injections for Limbs Spasticity: The Winning Duets
}

\author{
Abdallah RAHBANI ${ }^{1,2,3}$ and Francois Boulos ${ }^{2}$ \\ 1. Departement of Neurology, Lebanese Hospital university hospital Geitawi, 1100 Achrahieh Beirut, Lebanon \\ 2. Holy Spirit University, Faculty of Medical Sciences, 446 Jounieh, Mount Lebanon, Lebanon \\ 3. Lebanese University, Faculty of Medical Sciences, 1003 HadathBaabda, Mount Lebanon, Lebanon
}

\begin{abstract}
Botulinum toxin found its way into a lot of medical treatments improving the quality of life in many clinical situations. When treating spasticity, this toxin is injected in the affected muscle to decrease its tension and hyperactivity. The isolation of the affected muscles for injection is done either by anatomical palpation of the muscle which is also known as non-guided injection technique, or by using the ultrasound or the electromyography also known guided injection technique. In this study which is a prospective observational study that included patients over a 6 months period, 28 patients aged between 2 and 78 years, with spasticity naïve to botulinum toxin injections or had already been injected more than 3 months ago, were injected using guided injection technique. The optimal doses of botulinum toxin were administered based on the age, the severity of the condition, and affected muscle groups. In pediatric population these were also calculated according to the body weight. All patients were evaluated pre and 4 weeks post injection using the MAS Score (Modified Ashworth Scale). Statistical analysis showed a very significant response to treatment as compared to the pre-injection condition, for upper limbs, lower limbs, and all muscle groups with almost absent significant local or systemic side effects (with a $p$ value less than 0.001). Conclusion: guided techniques for botulinum toxin injections showed a great efficacy in the treatment of spasticity along with no regional or systemic side effects and are relatively well tolerated by patients. This finding leads to a better management of spasticity and to a decrease in oral medication intake and secondarily a decrease in their possible side effects.
\end{abstract}

Key words: Botulinum toxin, spasticity, EMG, ultrasound.

\section{Overview}

After the discovery of its possible therapeutic uses, the lethal botulinum neurotoxin rapidly took place in several treatments for a wide variety of diseases, improving the quality of life in patients dealing with chronic conditions. The toxin use has also spread widely in other non-therapeutic fields to become so popular in the aesthetic field with a huge demand in the market [1].

When dealing with spasticity, a lot of treatments, pharmacological or surgical, were studied. They all do have side effects. No treatment showed a local effect with relatively lower side effects as much as botulinum toxin did. Botulinum toxininjection is done

Corresponding author: Abdallah RAHBANI, MD, research field: neurology neurophysiology. either via guided or non-guided techniques [2]. The most popular and easier to do is the anatomical non-guided blind injection done by palpating the tense muscle and injecting it with the botulinum toxin.

Another injection technique is the guided technique. It is mainly done using electromyography (EMG) or ultrasound (US) [3, 4]. These techniques allow a better isolation of the affected muscle which leads us to better precision for injections.

The severity of spasticity is measured using the Modified Ashworth Scale (MAS). This scale is used to quantify the severity of the condition and to follow on its progression after the treatment and over time. Other scales might be used but are not included in this study.

The objective of our study is to demonstrate the efficacy and tolerability of guided botulinum toxin 
injection. These injections are performed using either EMG or ultrasound technique, which allows better isolation of the affected muscle leading to a more targeted procedure.

\section{Materials and Methods}

Thirty-one patients with limbs spasticity were injected with botulinum toxin at the Neurophysiology division between August and December 2017. These were naïve to treatment with botulinum toxin or had been already injected since more than 3 months without guidance. However only 28 patients were included in the study and presented to the follow-up visit 4 weeks following injection. Therefore 28 patients including 50 limbs and 94 muscle groups were injected under EMG or ultrasound guidance. All cases were evaluated using the Modified-Ashworth Scale (MAS) which was performed directly before and 4 weeks following the guided injections. From these patients, 25 patients were injected under EMG guidance and only 3 patients under ultrasound guidance. A "Nihon Kohden" machine with quantitative EMG software and TECA "myoject Luer Lock” Disposable hypodermic needles (Natus medical neurology incorporated) were used to locate and inject the affected muscle groups allowing a more precise targeting. For ultrasound guided technique a "Philips Affinity 50” machine was used. Onabotulinum Toxin A (Botox) [5] or Abobotulinum Toxin A (Dysport) [6] were used according to the indications and availability. For muscle injections using the EMG technique, the muscle activity was studied at rest upon minimal exertion, which allows the isolation of the over active muscle under EMG monitoring. Patients were informed about the possible local and systemic side effects of Botulinum toxin and a written consent was obtained in this setting. Doses of muscle relaxants (when prescribed) were maintained stable during the observation period. Table 1 summarizes patients' characteristics and groups of muscle injected.

\section{Results}

When evaluating four weeks following injection, there has been a significant decrease in the MAS score. With a confidence interval of $95 \%$ and a margin of error of $5 \%$, the data were analyzed with the help of SPSS software (Statistical Package for Social Science) version 20.

Descriptive statistics were calculated using percentages for qualitative variables and mean \pm standard deviation for quantitative variables to analyze baseline characteristics of study participants. The evolution of the score (for the lower and upper limbs) was calculated using the nonparametric pre post-test ( $k$ Related sample $T$ test: WILCOXON TEST). The decrease in the score was significant for almost all injected muscle groups, and for both upper and lower limbs. Table 2 shows the correspondent results for specific muscle groups. As per side effects and tolerability, there has been only one small muscle hematoma following one gastrocnemius muscle injection, that spontaneously resolved. No other complications were noted. The procedures were well tolerated by the patients.

Fig. 1 shows the difference in the MAS score pre and post injection of botulinum toxin for upper limbs muscles. Based on this variation, we can see the effect of the treatment by showing a decrease in the score reflecting an improvement in the condition of these patients.

Fig. 2 shows the difference in the MAS score pre and postinjection of botulinum toxin for lower limbs muscles. Based on this variation, we can see the effect of the treatment by showing a decrease in the score reflecting an improvement in the condition of these patients. 
Table 1 Patients characteristics, numbers and groups of muscles injected.

\begin{tabular}{|c|c|c|c|c|c|c|c|}
\hline Patient & Etiology & $\begin{array}{l}\text { Number of } \\
\text { affected limbs }\end{array}$ & $\begin{array}{l}\text { Preinjection MAS } \\
\text { (muscle groupscore) }\end{array}$ & $\begin{array}{l}\text { Post-injection MAS } \\
\text { (muscle groupscore) }\end{array}$ & Method & Age & Sex \\
\hline 1 & Perinatal stroke & $1 \mathrm{UE}$ & W2 F2 E2 & W1 F1 E0 & Ultrasound & 22 & M \\
\hline 2 & Ischemic stroke & $1 \mathrm{UE} 1 \mathrm{LE}$ & W3 F3 E2 GS2 & W2 F2 E2 GS1 & EMG & 78 & M \\
\hline 3 & Ischemic stroke & $1 \mathrm{UE}$ & W2 F2 E2 & W1 F1 E1 & EMG & 73 & M \\
\hline 4 & $\begin{array}{l}\text { Intracranial } \\
\text { Hemorrhage }\end{array}$ & $2 \mathrm{LE}$ & GS3 GS3 & $\begin{array}{l}\text { GS1+ } \\
\text { GS1+ }\end{array}$ & EMG & 58 & $\mathrm{~F}$ \\
\hline 5 & $\begin{array}{l}\text { Intracranial } \\
\text { Hemorrhage }\end{array}$ & $1 \mathrm{UE} 1 \mathrm{LE}$ & W4 F4 E3 GS3 & W4 F3 E1 GS1+ & EMG & 65 & $\mathrm{~F}$ \\
\hline 6 & Meningeal bleed & $1 \mathrm{UE}$ & W2 E2 & W1 E1 & EMG & 52 & F \\
\hline 7 & Perinatal stroke & $1 \mathrm{UE}$ & W3 F3 E2 & W2 F2 E1 & Ultrasound & 18 & $\mathrm{~F}$ \\
\hline 8 & Perinatal anoxia & $2 \mathrm{LE}$ & GS2 GS2 GRA1 & GS1 GS1 GRA0 & EMG & 3 & $\mathrm{~F}$ \\
\hline 9 & Ischemic stroke & $1 \mathrm{UE} 1 \mathrm{LE}$ & W3 F3 E2 GS2 & W2 F2 E1 GS1 & EMG & 70 & M \\
\hline 10 & Perinatal anoxia & $2 \mathrm{LE}$ & GS2 GS2 & GS1 GS1 & EMG & 2.5 & $\mathrm{~F}$ \\
\hline 11 & Perinatal anoxia & $2 \mathrm{LE}$ & GS2 GS2 GRA2 & GS1 GS1 GRA1 & EMG & 4 & M \\
\hline 12 & Meningeal bleed & $1 \mathrm{LE}$ & GS2 KE2 & GS1 KE1 & EMG & 64 & $\mathrm{~F}$ \\
\hline 13 & Ischemic stroke & $1 \mathrm{UE} 1 \mathrm{LE}$ & W3 F3 E2 GS2 & W2 F2 E1 GS1 & EMG & 67 & M \\
\hline 14 & Perinatal stroke & $1 \mathrm{UE}$ & W2 F2 E2 & W1 F1 E1 & EMG & 18 & $\mathrm{~F}$ \\
\hline 15 & Multiple sclerosis & $2 \mathrm{LE}$ & GS2 GS2 KE2 & GS1 GS1 KE1 & EMG & 42 & F \\
\hline 16 & Ischemic stroke & $1 \mathrm{UE}$ & W3 F3 E2 & W2 F2 E1 & EMG & 58 & M \\
\hline 17 & Cervical myelopathy & $2 \mathrm{LE}$ & GS3 GS3 KE2 KE2 & GS2 GS2 KE1 KE1 & EMG & 56 & $\mathrm{~F}$ \\
\hline 18 & Multiple sclerosis & $1 \mathrm{UE} 1 \mathrm{LE}$ & W2 F2 E2 GS2 & W1+ F1 E1 GS1 & EMG & 52 & M \\
\hline 19 & Perinatal anoxia & $2 \mathrm{LE}$ & GS2 GS2 GRA1 & GS1 GS1 GRA0 & EMG & 4 & $\mathrm{~F}$ \\
\hline 20 & Ischemic stroke & $1 \mathrm{UE}$ & W2 F3 E2 & W1 F1+ E1 & Ultrasound & 63 & $\mathrm{~F}$ \\
\hline 21 & Ischemic stroke & $1 \mathrm{UE}$ & W2 F1 E2 & W1 F0 E1 & EMG & 57 & M \\
\hline 22 & Multiple sclerosis & $2 \mathrm{LE}$ & GS2 GS2 KE2 KE2 & GS1 GS1 KE1 KE1 & EMG & 36 & M \\
\hline \multirow[t]{2}{*}{23} & Hereditary & $2 \mathrm{LE}$ & GS2 GS2 & GS1 GS1 & EMG & 27 & $\mathrm{~F}$ \\
\hline & Spastic paraparesis & & GRA1+ GRA1+ & GRA0 GRA0 & & & \\
\hline 24 & Head trauma & $1 \mathrm{UE} 1 \mathrm{LE}$ & W2 F2 E2 GS1 & W1 F1+ E1 GS0 & EMG & 36 & M \\
\hline 25 & Perinatal anoxia & $2 \mathrm{LE}$ & $\begin{array}{l}\text { GS2 GS2 GRA2 } \\
\text { GRA2 } \\
\text { W2 W2 }\end{array}$ & $\begin{array}{l}\text { GS2 GS2 GRA2 } \\
\text { GRA2 } \\
\text { W1 W1 }\end{array}$ & EMG & 2 & M \\
\hline 26 & Multiple sclerosis & 2 UE 2 LE & $\begin{array}{l}\text { F2 F2 E1 } \\
\text { E1 KE2 KE2 }\end{array}$ & $\begin{array}{l}\text { F1 F1 E0 } \\
\text { E0 KE1 KE0 }\end{array}$ & EMG & 46 & $\mathrm{~F}$ \\
\hline 27 & Perinatal anoxia & $2 \mathrm{LE}$ & $\begin{array}{l}\text { GS2 GS2 } \\
\text { GRA1+ GRA1+ }\end{array}$ & $\begin{array}{l}\text { GS1 GS1 GRA0 } \\
\text { GRA0 GRA0 }\end{array}$ & EMG & 2.5 & M \\
\hline 28 & Perinatal anoxia & $2 \mathrm{LE}$ & GS2 GS2 & GS0 GS0 & EMG & 3 & M \\
\hline
\end{tabular}

UE = Upper extremity, LE = Lower extremity, M = Male, F = Female, GS = Gastrocnemius soleus complex, KE = Knee extensors complex, $\mathrm{W}=$ Wrist flexors, $\mathrm{F}=$ Finger flexors, $\mathrm{E}=$ Elbow flexors, GRA = Gracilis/adductors complex.

Table 2 MAS scores before and 4 weeks following Botulinum toxin injections.

\begin{tabular}{lllc}
\hline Variable dependent & Pre-score & Post-score & $p$ value \\
\hline Wrist flexors & $2.6 \pm 0.734$ & $1.53 \pm 0.834$ & $0.0001^{*}$ \\
Elbow flexors & $2.07 \pm 0.258$ & $0.87 \pm 0.352$ & $0.0001^{*}$ \\
Finger flexors & $2.64 \pm 0.842$ & $1.50 \pm 0.76$ & $0.0001^{*}$ \\
Gastrocnemius soleus complex & $3.21 \pm 1.228$ & $1.58 \pm 1.228$ & $0.0001^{*}$ \\
Knee extensors complex & $2.6 \pm 1.34$ & $1.8 \pm 0.837$ & 0.178 \\
Gracilis/adductors complex & $1.33 \pm 0.516$ & $0.17 \pm 0.40$ & $0.0001^{*}$ \\
\hline
\end{tabular}

Quantitative variables are expressed by mean \pm standard deviation;

* Indicates a significant difference between the two groups with a value of $p<0.05$. 


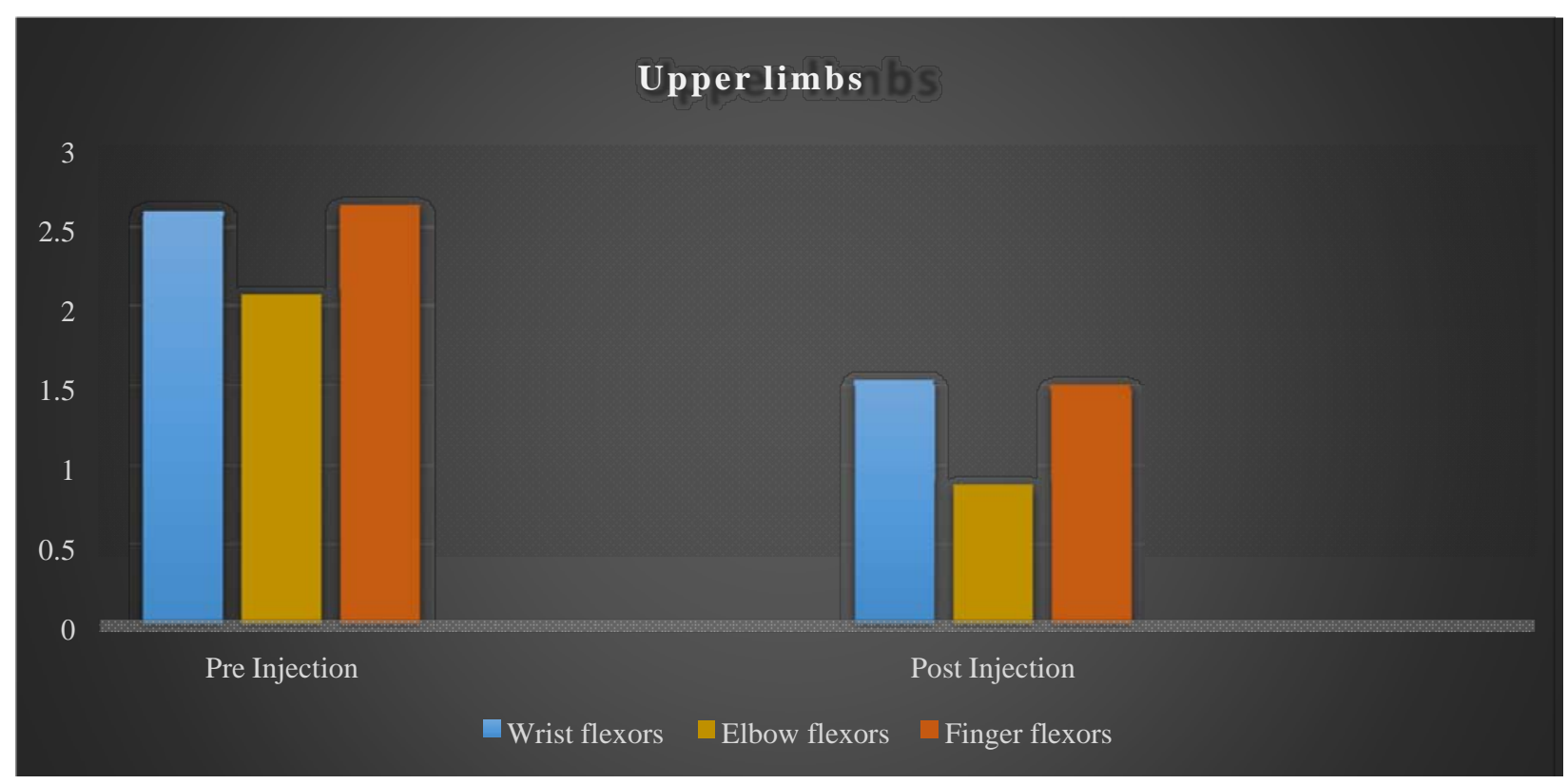

Fig. 1 The degree of improvement in upper limb spasticity comparing all the muscle groups.

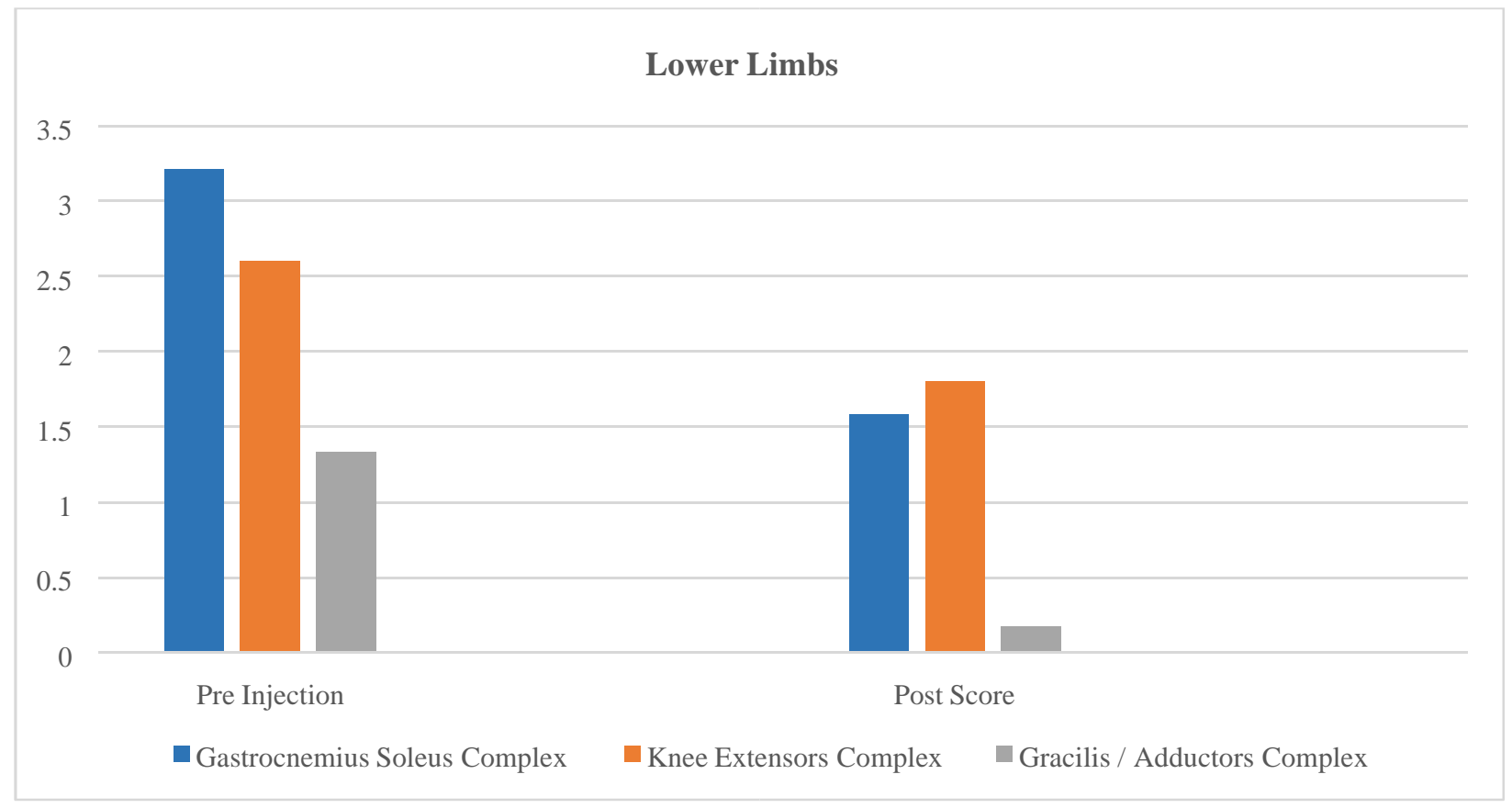

Fig. 2 The degree of improvement in lower limb spasticity comparing all the muscle groups.

\section{Discussion}

Botulinum toxin injection represents a relatively safe treatment for spasticity. When administered correctly using the optimal dose, injected in specified targets, it can decrease spasticity enormously with relatively low risk of side effects, adjacent structures' damage, and local limb weakness. This beneficial treatment can present sometimes with local or regional weakness. It is especially seen in blind anatomical localization for injection of spastic muscle groups which is not here the case.

Both EMG and Ultrasound allow a clear identification and isolation of the affected muscles. 
The Ultrasound, by reproducing the image of the underneath affected muscle, and the EMG, by isolating the hyperactive muscle at rest, and after activation. These two techniques provide the physician with a clearer view of the targeted muscle making the treatment well defined and more precise.

Four weeks following injection, patients showed remarkable clinical benefit both for upper and lower limbs spasticity and for all groups of muscles injected, independently from the type of toxin injected (Botox or Dysport). The improvement was statistically very significant with a $p$ value of 0.001 . This was associated with secondary improvement of the quality of life, the performance of activities of daily living in addition to relative decrease in pain sensation, but accurate statistical data are not available for these parameters.

Both adults and children presented beneficial effect of the treatment as demonstrated by the above results.

The results we have got concerning the efficacy of the treatment were similar to results obtained in many studies [7, 8] stating the superiority of the guided injection techniques over the non-guided injection techniques.

Guided injection techniques showed better results over the non-guided injection techniques. The ultrasound technique showed a better definition of the targeted muscle, with a finest precision in muscle isolation and treatment. A similar efficacy was seen by using the EMG technique. The same techniques used in our study gave as satisfying results, the same as results of other conducted studies. The validity of the score along with the high efficacy of the treatment demonstrated by the high significance of the statistical analysis, adding the lack of side effects and the good tolerability of the treatment, offer for this study a good basis.

Some limitations of our results are noted and included mostly the lack of comparative data analysis concerning the efficacy of the guided injections over the blind anatomical injections mostly for the subgroup of patient's who initially failed nonguided injections (12 patients).Another point is the inability to compare the degree of improvement in those cases treated under ultrasound versus those treated under EMG guidance due to the small number of the former cases and a larger study appears to be necessary in order to obtain the expected information.

\section{Conclusion}

Adding the theory to the practice, this study succeeded in determining a statistical evidence stating that the guided botulinum toxin injection has a clear benefit and an excellent side effects profile in the treatment of spasticity that apparently is higher than the non-guided blind anatomical injections even in the absence of comparative data.

Given this data and following this study, by guiding the injections, the toxin could be held into the muscle without affecting adjacent structures. This can lead us to decrease the risk of excessive limb weakness along with other regional and systemic side effect, making this treatment for spasticity relatively safer than other therapeutical strategies where more side effects are observed.

By injecting patients using the guided techniques, the quantity of botulinum toxin needed for injections is usually lesser and the effect of the treatment is higher, limiting treatment related side effects and delivering better results to patients suffering from spasticity. Therefore, greater clinical improvement and the ability to reduce the amount of oral medications used for treatment of muscle pain and spasms are observed.

\section{References}

[1] Erbguth, F. J. 2008. "From Poison to Remedy: The Chequered History of Botulinum Toxin.” Journal of Neural Transmission 115 (4): 559-65. https://doi.org/10.1007/s00702-007-0728-2.

[2] Walker, H. W., Lee, M. Y., Bahroo, L. B., Hedera, P., and Charles, D. 2015. "Botulinum Toxin Injection Techniques for the Management of Adult Spasticity.” PM \& R: The Journal of Injury, Function, and Rehabilitation 
7 (4): 417-27. https://doi.org/10.1016/j.pmrj.2014.09.021

[3] Medica, E. M., Buyukavci, R., and Akturk, S. 2018.

"Evaluating the Functional Outcomes of Ultrasoundguided Botulinum Toxin Type A Injections Using the Euro-musculus Approach for Upper Limb Spasticity Treatment in Post-stroke Patients: An Observation." European Journal of Physical and Rehabilitation Medicine. https:/doi. org/10.23736/S1973-9087.18.05086-4.

[4] Santamato, A., Micello, M. F., Panza, F., Fortunato, F., Baricich, A., Cisari, C., et al. 2014. "Can Botulinum Toxin Type A Injection Technique Influence the Clinical Outcome of Patients with Post-stroke Upper Limb Spasticity? A Randomized Controlled Trial Comparing Manual Needle Placement and Ultrasound-guided Injection Techniques.” Journal of the Neurological Sciences $\quad 347 \quad(1-2)$ : $39-43$. https://doi.org/10.1016/j.jns.2014.09.016.
[5] ALLERGAN. 2017.2 "BOTOX (OnabotulinumtoxinA) for Injection, for Intramuscular, Intradetrusor, or Intradermal Use.” FDA.

[6] IPSEN. 2009. "DYSPORT ${ }^{\circledR}$ (AbobotulinumtoxinA) for Injection, for Intramuscular Use.” FDA.

[7] Jiang, L., Dou, Z.-L., Wang, Q., Wang, Q.-Y., Dai, M., Wang, Z., et al. 2015. "Evaluation of Clinical Outcomes of Patients with Post-stroke Wrist and Finger Spasticity after Ultrasonography-guided BTX-A Injection and Rehabilitation Training." Frontiers in Human Neuroscience $\quad 9 \quad$ (September): https://doi.org/10.3389/fnhum.2015.00485.

[8] Marciniak, C., et al, 2017. Efficacy and Safety of AbobotulinumtoxinA (Dysport) for the Treatment of Hemiparesis in Adults With Upper Limb Spasticity Previously Treated With Botulinum Toxin: Subanalysis From a Phase 3 Randomized Controlled Trial.” PMR 9 (12): 1181-90. https://doi: 10.1016/j.pmrj.2017.06.007. 